\title{
Discovery and Atmospheric Characterization of Giant Planet Kepler-12b: An Inflated Radius Outlier
}

\section{Citation}

Fortney, Jonathan J., Brice-Olivier Demory, Jean-Michel Désert, Jason Rowe, Geoffrey W. Marcy, Howard Isaacson, Lars A. Buchhave, et al. 2011. "DISCOVERY AND ATMOSPHERIC CHARACTERIZATION OF GIANT PLANET KEPLER-12b: AN INFLATED RADIUS OUTLIER." The Astrophysical Journal Supplement Series 197 (1): 9. https://doi.org/10.1088/0067-0049/197/1/9.

\section{Permanent link}

http://nrs.harvard.edu/urn-3:HUL.InstRepos:41397445

\section{Terms of Use}

This article was downloaded from Harvard University's DASH repository, and is made available under the terms and conditions applicable to Other Posted Material, as set forth at http:// nrs.harvard.edu/urn-3:HUL.InstRepos:dash.current.terms-of-use\#LAA

\section{Share Your Story}

The Harvard community has made this article openly available. Please share how this access benefits you. Submit a story.

Accessibility 


\title{
DISCOVERY AND ATMOSPHERIC CHARACTERIZATION OF GIANT PLANET KEPLER-12b: AN INFLATED RADIUS OUTLIER
}

\author{
Jonathan J. Fortney ${ }^{1,21}$, Brice-Olivier Demory ${ }^{2}$, Jean-Michel Désert ${ }^{3}$, Jason Rowe ${ }^{4}$, Geoffrey W. Marcy ${ }^{5}$, \\ Howard Isaacson $^{5}$, Lars A. Buchhave ${ }^{3,6}$, David Ciardi ${ }^{7}$, Thomas N. Gautier ${ }^{8}$, Natalie M. Batalha ${ }^{9}$, Douglas \\ A. Caldwell ${ }^{4}$, Stephen T. Bryson ${ }^{10}$, Philip Nutzman ${ }^{1}$, Jon M. Jenkins ${ }^{4}$, Andrew Howard ${ }^{5}$, David Charbonneau ${ }^{3}$, \\ Heather A. Knutson ${ }^{5}$, Steve B. Howell ${ }^{10}$, Mark Everett ${ }^{11}$, François Fressin $^{3}$, Drake Deming ${ }^{12}$, William J. Borucki ${ }^{10}$, \\ Timothy M. Brown ${ }^{13}$, Eric B. Ford ${ }^{14}$, Ronald L. Gilliland ${ }^{15}$, David W. Latham ${ }^{3}$, Neil Miller ${ }^{1}$, Sara Seager $^{2}$, \\ Debra A. Fischer ${ }^{16}$, David Koch ${ }^{10}$, JACK J. Lissauer ${ }^{10}$, Michael R. HaAs ${ }^{10}$, Martin Still ${ }^{17}$, Philip LuCas ${ }^{18}$, \\ Michael Gillon ${ }^{19,20}$, Jessie L. Christiansen ${ }^{4}$, And John C. Geary ${ }^{3}$ \\ ${ }^{1}$ Department of Astronomy and Astrophysics, University of California, Santa Cruz, CA 95064, USA; jfortney @ ucolick.org \\ ${ }^{2}$ Massachusetts Institute of Technology, Cambridge, MA 02139, USA \\ ${ }^{3}$ Harvard-Smithsonian Center for Astrophysics, 60 Garden Street, Cambridge, MA 02138, USA \\ ${ }^{4}$ SETI Institute/NASA Ames Research Center, Moffett Field, CA 94035, USA \\ ${ }^{5}$ Department of Astronomy, University of California, Berkeley, CA 94720-3411, USA \\ ${ }^{6}$ Niels Bohr Institute \& StarPlan, University of Copenhagen, Copenhagen, Denmark \\ ${ }^{7}$ NASA Exoplanet Science Institute, Caltech, MS 100-22, 770 South Wilson Avenue, Pasadena, CA 91125, USA \\ 8 Jet Propulsion Laboratory/California Institute of Technology, Pasadena, CA 91109, USA \\ ${ }^{9}$ Department of Physics and Astronomy, San Jose State University, San Jose, CA 95192, USA \\ ${ }^{10}$ NASA Ames Research Center, Moffett Field, CA 94035, USA \\ ${ }^{11}$ National Optical Astronomy Observatories, Tucson, AZ 85719, USA \\ 12 Department of Astronomy, University of Maryland at College Park, College Park, MD 20742, USA \\ ${ }^{13}$ Las Cumbres Observatory Global Telescope, Goleta, CA 93117, USA \\ ${ }^{14}$ Department of Astronomy, University of Florida, Gainesville, FL 32611, USA \\ ${ }^{15}$ Space Telescope Science Institute, Baltimore, MD 21218, USA \\ ${ }^{16}$ Department of Astronomy, Yale University, New Haven, CT 06520, USA \\ ${ }^{17}$ Bay Area Environmental Research Institute/NASA Ames Research Center, Moffett Field, CA 94035, USA \\ ${ }^{18}$ Centre for Astrophysics Research, University of Hertfordshire, College Lane, Hatfield AL10 9AB, UK \\ ${ }^{19}$ Institut d'Astrophysique et de Géophysique, Université de Liège, Allée du 6 Août 17, Bat. B5C, 4000 Liège, Belgium \\ ${ }^{20}$ Observatoire de Genève, Université de Genève, 51 Chemin des Maillettes, 1290 Sauverny, Switzerland \\ Received 2011 July 7; accepted 2011 September 29; published 2011 October 26
}

\begin{abstract}
We report the discovery of planet Kepler-12b (KOI-20), which at $1.695 \pm 0.030 R_{\mathrm{J}}$ is among the handful of planets with super-inflated radii above $1.65 R_{\mathrm{J}}$. Orbiting its slightly evolved G0 host with a 4.438 day period, this $0.431 \pm 0.041 M_{\mathrm{J}}$ planet is the least irradiated within this largest-planet-radius group, which has important implications for planetary physics. The planet's inflated radius and low mass lead to a very low density of $0.111 \pm 0.010 \mathrm{~g} \mathrm{~cm}^{-3}$. We detect the occultation of the planet at a significance of $3.7 \sigma$ in the Kepler bandpass. This yields a geometric albedo of $0.14 \pm 0.04$; the planetary flux is due to a combination of scattered light and emitted thermal flux. We use multiple observations with Warm Spitzer to detect the occultation at $7 \sigma$ and $4 \sigma$ in the 3.6 and $4.5 \mu \mathrm{m}$ bandpasses, respectively. The occultation photometry timing is consistent with a circular orbit at $e<0.01$ $(1 \sigma)$ and $e<0.09(3 \sigma)$. The occultation detections across the three bands favor an atmospheric model with no dayside temperature inversion. The Kepler occultation detection provides significant leverage, but conclusions regarding temperature structure are preliminary, given our ignorance of opacity sources at optical wavelengths in hot Jupiter atmospheres. If Kepler-12b and HD 209458b, which intercept similar incident stellar fluxes, have the same heavy-element masses, the interior energy source needed to explain the large radius of Kepler-12b is three times larger than that of HD 209458b. This may suggest that more than one radius-inflation mechanism is at work for Kepler-12b or that it is less heavy-element rich than other transiting planets.
\end{abstract}

Key words: planets and satellites: atmospheres - stars: individual (Kepler-12, KOI-20, KIC 11804465) techniques: spectroscopic

Online-only material: color figures

\section{INTRODUCTION}

Transiting planets represent an opportunity to understand the physics of diverse classes of planets, including mass-radius regimes not found in the solar system. The knowledge of the mass and radius of an object immediately yields the bulk density, which can be compared to models to yield insight into the planet's internal composition, temperature, and

\footnotetext{
${ }^{21}$ Alfred P. Sloan Research Fellow.
}

structure (e.g., Miller \& Fortney 2011). Subsequent observations at the time of the planet's occultation (secondary eclipse) allow for the detection of light emitted or scattered by the planet's atmosphere, which can give clues to a planet's dayside temperature structure and chemistry (Marley et al. 2007; Seager $\&$ Deming 2010). NASA's Kepler Mission was launched on 2009 March 7 with the goal of finding Earth-sized planets in Earth-like orbits around Sun-like stars (Borucki et al. 2010). While working toward this multi-year goal, it is also finding an 


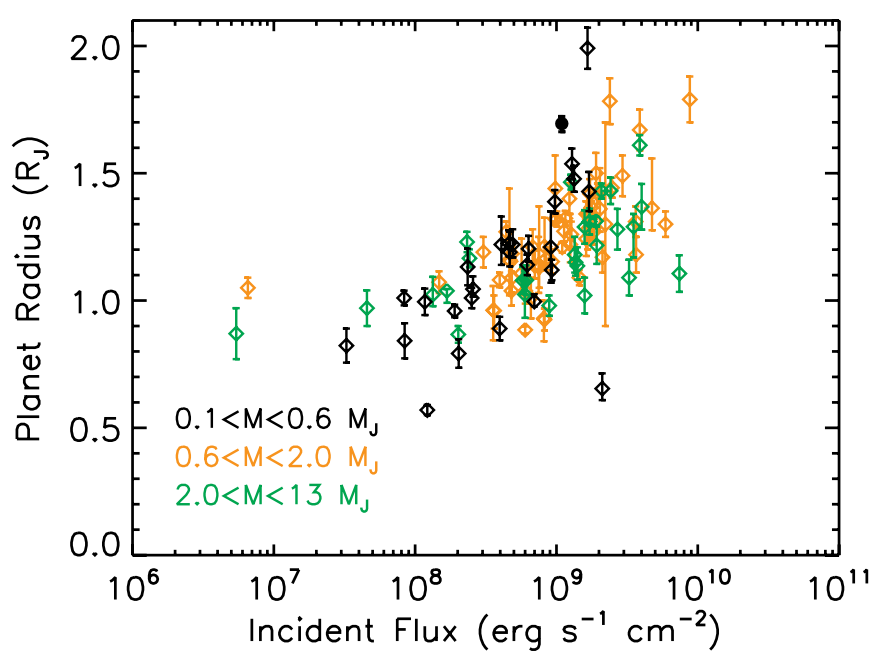

Figure 1. Observed planetary radius as a function of total incident flux for the known transiting gas giants. Planets are plotted in three colors for three different mass cuts. Kepler-12b is shown as a black filled circle. Note the general trend toward smaller radii with decreasing insolation. There is a probable break in slope at incident fluxes of $1-2 \sim 10^{8} \mathrm{erg} \mathrm{cm}^{-2} \mathrm{~s}^{-1}$. Planets are taken from the compilation at http://www.inscience.ch/transits/.

(A color version of this figure is available in the online journal.)

interesting menagerie of larger and hotter planets that are aiding our understanding of planetary physics.

Early on in the mission, follow-up radial velocity (RV) resources preferentially went to giant planets for which it would be relatively easy to confirm their planetary nature through a measurement of planetary mass. This is how the confirmation of planet Kepler-12b was made, at first glance a relatively standard "hot Jupiter" in a 4.438 day orbit. However, upon further inspection, the mass and radius of Kepler-12b make it an interesting planet from the standpoint of the now-familiar "radius anomaly" of transiting giant planets (e.g., Charbonneau et al. 2007; Burrows et al. 2007; Laughlin et al. 2011). Given our current understanding of strongly irradiated giant planet thermal evolution, around $1 / 3$ to $1 / 2$ of known transiting planets are larger than models predict for several-gigayear-old planets that cool and contract under intense stellar irradiation (Miller et al. 2009).

The observation that many Jupiter- and Saturn-mass planets are larger than 1.0 Jupiter-radii can be readily understood. It is the magnitude of the effect that still needs explanation. The first models of strongly irradiated planets yielded the prediction that these close-in planets would be inflated in radius compared to Jupiter and Saturn (Guillot et al. 1996). The high incident flux drives the radiative convective boundary from less than a bar, as in Jupiter, to pressures near a kilobar. The thick radiative zone transports less flux than a fully convective atmosphere, thereby slowing interior cooling, which slows contraction. A fairly uniform prediction of these strongly irradiated models is that $1.2-1.3 R_{\mathrm{J}}$ is about the largest radii predicted for planets several gigayears old (Bodenheimer et al. 2003; Burrows et al. 2007; Fortney et al. 2007; Baraffe et al. 2008). However, planets commonly exceed this value.

The mechanism that leads to the radius anomaly has not yet been definitively identified. However, constraints are emerging. One is planet radius versus incident flux, which could also be thought of as radius versus equilibrium temperature, with an assumption regarding planetary Bond albedos. Figure 1 shows planet radii versus incident flux for the transiting systems with confirmed masses. Since low-mass planets are relatively easier to inflate to large radii than higher mass planets (e.g., Miller et al. 2009), we plot the planets in three mass bins. The lowest mass bin is Saturn-like masses, while the middle mass bin is Jupiter-like masses. The upper mass bin ends at $13 M_{\mathrm{J}}$, the deuterium burning limit. Kepler-12b is shown as a black filled circle. The largest radius planets are generally the most highly irradiated (Kovács et al. 2010; Laughlin et al. 2011; Batygin et al. 2011). The near-universality of the inflation, especially at high incident fluxes, now clearly argues for a mechanism that affects all close-in planets (Fortney et al. 2006), rather than one that affects only some planets. The distribution of the radii could then be understood in terms of differing magnitudes of the inflation mechanism, together with different abundances of heavy elements within the planets (Fortney et al. 2006; Guillot et al. 2006; Burrows et al. 2007; Miller \& Fortney 2011; Batygin et al. 2011).

Within this emerging picture, outlier points are particularly interesting: those that are especially large, given their incident flux. These are the super-inflated planets with radii of $1.7 R_{\mathrm{J}}$ or larger. These include WASP-12b (Hebb et al. 2009), TrES-4b (Mandushev et al. 2007; Sozzetti et al. 2009), WASP-17b (Anderson et al. 2010), and now Kepler-12b, which is the least irradiated of the four. In the following we describe the discovery of Kepler-12b, along with the initial characterization of the planet's atmosphere.

Transiting planets enable the characterization of exoplanet atmospheres. The Spitzer Space Telescope has been especially useful for probing the dayside temperature structure of close-in planetary atmospheres, as thermal emission from the planets can readily be detected by Spitzer at wavelengths longer than $3 \mu \mathrm{m}$. Data sets are becoming large enough that one can begin to search for correlations in the current detections (Knutson et al. 2010; Cowan \& Agol 2011).

A powerful new constraint of the past two years is the possibility of joint constraints in the infrared, from Spitzer, and the optical, from space telescopes like CoRoT (e.g., Gillon et al. 2010; Deming et al. 2011) and Kepler (Désert et al. 2011a). The leverage from optical wavelengths comes from a measurement (or upper limit) of the geometric albedo of the planet's atmosphere, although this is complicated by a mix of thermal emission and scattered light both contributing for these planets. Detection of relatively low geometric albedos $A_{\mathrm{g}}<0.15$ is consistent with cloud-free models of hot Jupiter atmospheres (Sudarsky et al. 2003; Burrows et al. 2008), and can inform our understanding of what causes the temperature inversions in many hot Jupiter atmospheres (Spiegel \& Burrows 2010).

In this paper, we discuss all aspects of the detection, validation, confirmation, and characterization of the planet. Section 2 discusses the detection of the planet by Kepler, while Section 3 covers false-positive rejection and RV confirmation. Section 4 gives the global fit to all data sets to derive stellar and planetary parameters, while Section 5 concerns the observational and modeling aspects of atmospheric characterization. Section 6 is a discussion of the planet's inflated radius amongst its peers, while Section 7 gives our conclusions.

\section{DISCOVERY}

The Kepler science data for the primary transit search mission are the long cadence (LC) data (Jenkins et al. 2010b). These consist of sums close to 30 minutes of each pixel in the aperture containing the target star in question. These data proceed through an analysis pipeline to produce corrected pixel data, 

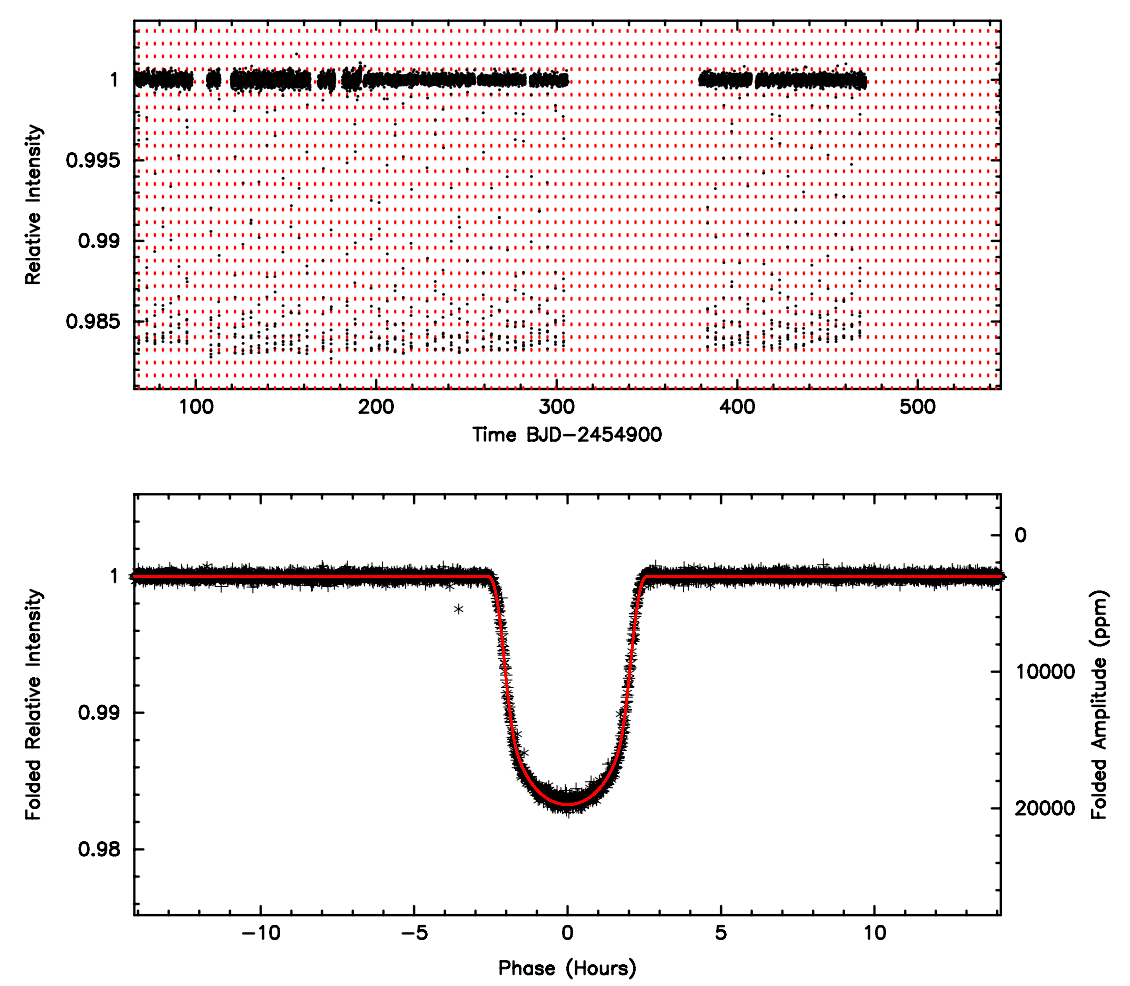

Figure 2. Time series and folded transit light curve for Kepler-12b.

(A color version of this figure is available in the online journal.)

then simple unweighted aperture photometry sums are formed to produce a photometric time series for each object (Jenkins et al. 2010c). The many thousands of photometric time series are then processed by the transiting planet search (TPS) pipeline element (Jenkins et al. 2010c).

The candidate transit events identified by TPS are also vetted by visual inspection. The light curves produced by the photometry pipeline tend to show drifts due to an extremely small, slow focus change (Jenkins et al. 2010c), and there are also sometimes low-frequency variations in the stellar signal that can make analysis of the transit somewhat problematic. These low-frequency effects can be removed by modest filters that have only an insignificant effect on the transit signal (Koch et al. 2010). The unfolded and folded light curves for Kepler-12b produced in this manner are shown in Figure 2.

Centroid analysis was performed using both difference image (Torres et al. 2011) and photocenter motion (Jenkins et al. 2010a) techniques using Q1 through Q4 data. This analysis indicates that the object with the transiting signal is within 0.01 pixels ( 0.04 arcsec) of Kepler-12, which is the $3 \sigma$ radius of confusion (including systematic biases) for these techniques.

The parent star, Kepler Input Catalog (KIC) identification number 11804465, has a magnitude in the Kepler band of 13.438. The KIC used ground-based multi-band photometry to assign an effective temperature and surface gravity of $T_{\text {eff }}=$ $6012 \mathrm{~K}$ and $\log g=4.47$ (cgs) to Kepler-12, corresponding to a late-F or early-G dwarf. Stellar gravities in this part of the H-R diagram are difficult to determine from photometry alone, and one of our conclusions based on high-resolution spectroscopy and light curve analyses in Section 4 is that the star is near the end of its main-sequence lifetime, with a radius that has expanded to $R_{*}=1.483 \pm 0.027 R_{\odot}$ and a surface gravity of $\log g=4.175 \pm 0.013$. In turn, this implies an inflated radius for the planet candidate, originally known as Kepler Object of
Interest (KOI)-20 (Borucki et al. 2011). This conclusion is hard to avoid, because the relatively long duration of the transit, more than $5 \mathrm{hr}$ from first to last contact, demands a low density and expanded radius for the star.

\section{CONFIRMATION: FOLLOW-UP OBSERVATIONS}

\subsection{High-resolution Imaging from Large Telescopes}

Blends due to unresolved stellar companions (associated or background) can only be ruled out with direct imaging from large telescopes. In Figure 3, we show an image of Kepler12 taken with the Keck I telescope guide camera, showing $9 \times 9$ arcsec taken in 0.8 arcsec seeing. This $1.0 \mathrm{~s}$ exposure was taken with a BG38 filter, making the passband roughly 400-800 $\mathrm{nm}$, similar to that of Kepler. Contours show surface brightness relative to the core. No companion is seen down to 7 mag fainter than Kepler-12 beyond $\sim 1$ arcsec from it. Thus, there is no evidence of a star that could be an eclipsing binary, consistent with the lack of astrometric displacement during transit.

In addition, speckle observations using the WIYN telescope were made on the night of 2010 June 18/19, as part of the Kepler follow-up program of S. Howell and collaborators (Howell et al. 2011). No additional sources were seen to $3.69 \mathrm{mag}$ fainter in $R$ band and 2.17 mag fainter in $V$ band in an annulus around the star spanning between 0.1 and 0.3 arcsec in radius. No companions could be seen as close as the diffraction limit $(0.05$ arcsec from the star) or as far as the edge of the $2.8 \times 2.8$ arcsec field of view.

Near-infrared adaptive optics imaging of Kepler-12 was obtained on the night of 2009 September 8 UT with the Palomar Hale 200 inch telescope and the PHARO near-infrared camera (Hayward et al. 2001) behind the Palomar adaptive optics system (Troy et al. 2000). PHARO, a $1024 \times 20124 \mathrm{HgCdTe}$ infrared array, was utilized in the 25.1 mas pixel $^{-1}$ mode yielding 


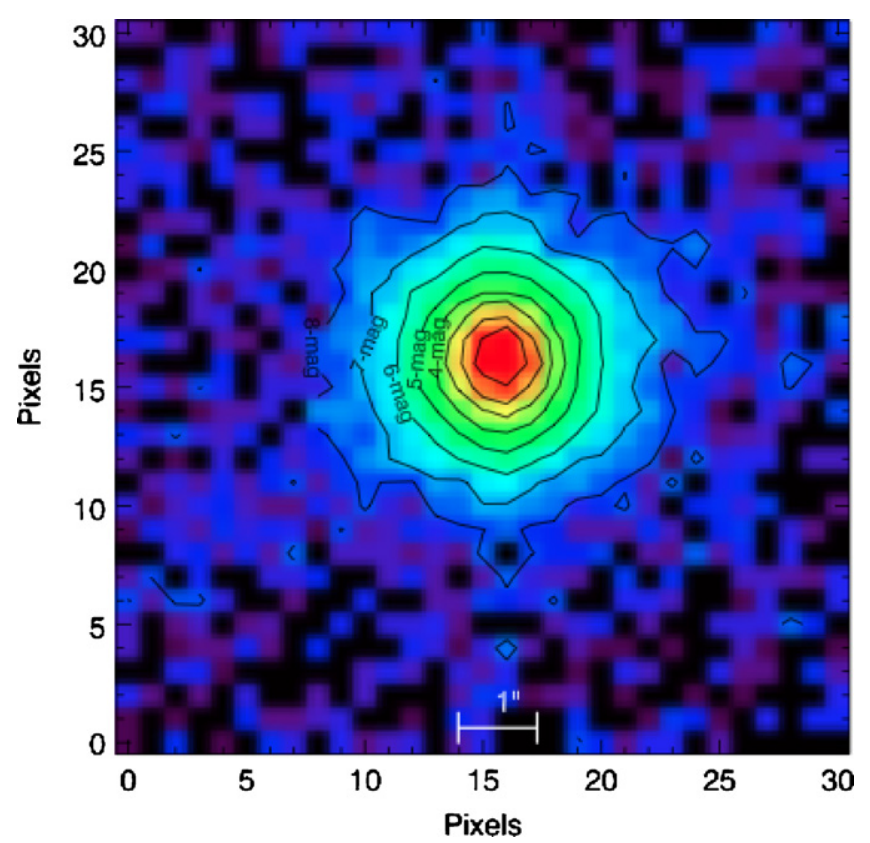

Figure 3. Image of Kepler-12 taken with the Keck I telescope guide camera, showing $9 \times 9$ arcsec taken in 0.8 arcsec seeing. North is up and east is to the left and the pixels are 0.30 arcsec in size. The exposure time was $1.0 \mathrm{~s}$. The detector is a Photometrics CCD and the filter is a BG38, making the passband roughly $400-800 \mathrm{~nm}$, similar to that of Kepler. Contours show surface brightness relative to the core. No companion is seen down to 7 mag fainter than Kepler-12 beyond $\sim 1$ arcsec from it. There is no evidence of a star that could be an eclipsing binary.

(A color version of this figure is available in the online journal.)

a field of view of $25^{\prime \prime}$. Observations were performed in $J$ filter $\left(\lambda_{0}=1.25 \mu \mathrm{m}\right)$. The data were collected in a standard 5 -point quincunx dither pattern of $5^{\prime \prime}$ steps interlaced with an off-source ( $60^{\prime \prime}$ East) sky dither pattern. Data were taken with integration times per frame of $60 \mathrm{~s}$ (15 frames) for a total onsource integration time of 15 minutes. The individual frames were reduced with a custom set of IDL routines written for the PHARO camera and were combined into a single final image. The adaptive optics system guided on the primary target itself and produced a central core width of FWHM $=0$ ". 11 . The final co-added image at $J$ is shown in Figure 4.

One additional source was detected at $5^{\prime \prime} \mathrm{SE}$ and $\Delta J \approx 8 \mathrm{mag}$ fainter than the primary target, near the limit of the observations. No additional sources were detected at $J$ within 7.5 of the primary target. Source detection completeness was evaluated by measuring the median level and dispersion within a series of annular rings, surrounding the primary target. Each ring has a width of 0 '. $11=1 \mathrm{FWHM}$, and each successive ring is stepped from the previous ring by $0.11=1$ FWHM. The median flux level and the dispersion of the individual rings were used to set the $4 \sigma$ sensitivity limit within each ring. The measured limits are in the $J$ band, but have been converted to limits in the Kepler bandpass based upon the typical $m_{\text {Kepler }}-J=1.28 \pm 0.52 \mathrm{mag}$ for a magnitude-limited sample (Howell et al. 2011). A summary of the detection efficiency as a function of distance from the primary star is given in Figure 5.

\subsection{Radial Velocity}

To derive the planetary mass and confirm the planetary nature of the companion, observations of the reflex motion of the Kepler-12b parent star were made. The line-of-sight RV variations of the parent star were made with the HIRES instrument (Vogt et al. 1994) on Keck I. Furthermore, a template spectrum observation was used to determine the stellar $T_{\text {eff }}$, metallicity, and the initial $\log g$, using the Spectroscopy Made Easy (SME) tools. The $\log g$ value from spectroscopy was $4.15 \pm 0.05$, considerably lower from the value in the KIC (4.47), but in good agreement with the value obtained from the Markov Chain Monte Carlo (MCMC) analysis described in Section 4 . The determined $T_{\text {eff }}$ is $5947 \pm 100 \mathrm{~K}$, with a distance estimate of $\sim 600 \mathrm{pc}$. We note that the star is chromospherically very quiet. Our HIRES spectra cover the CaII H\&K lines, and we measure a chromospheric index, $S=0.128$ and $\log R_{\mathrm{HK}}^{\prime}=$ -5.25 , indicating very low magnetic activity, consistent with an old, slowly rotating star.

All but the last four RVs were obtained during the first followup season, during the summer of 2009. The early Keck-HIRES spectra were taken with two compromising attributes. With a
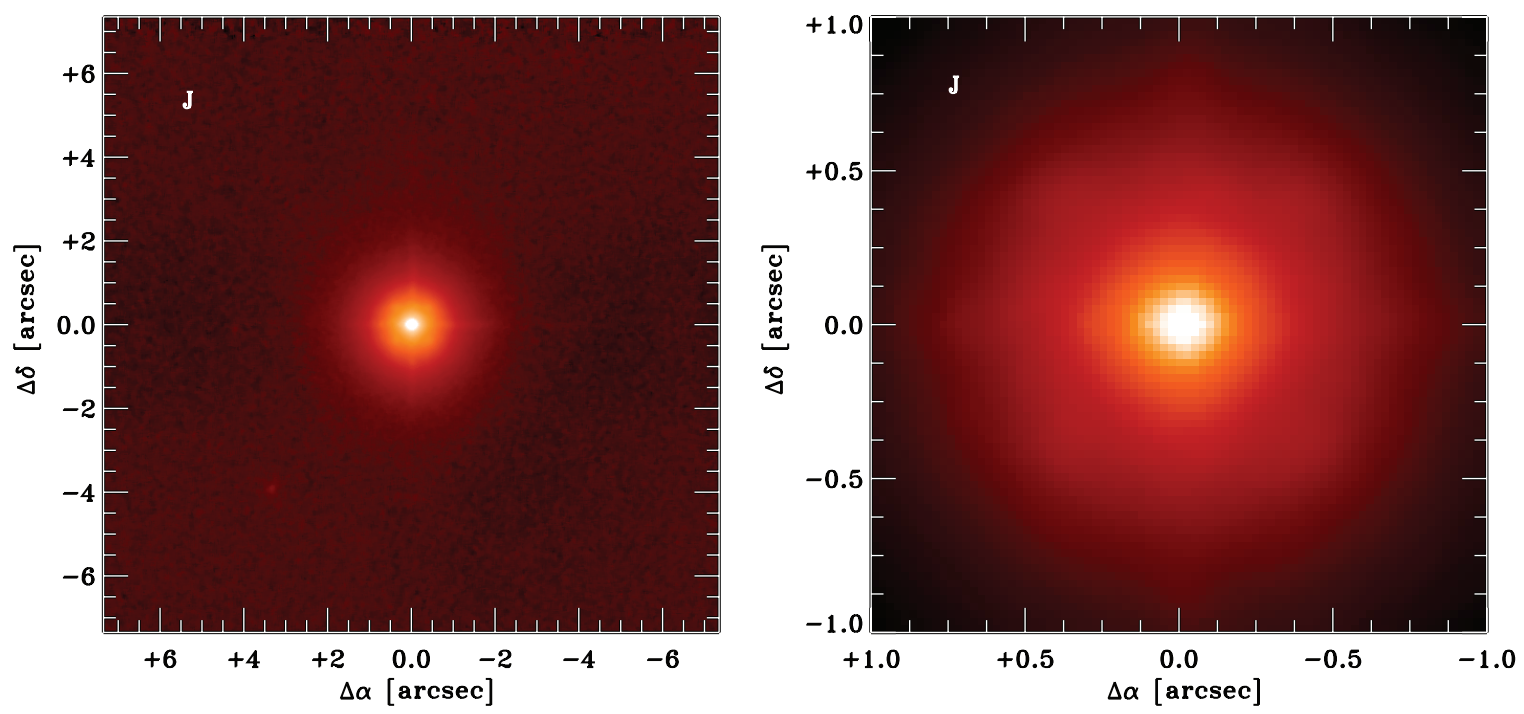

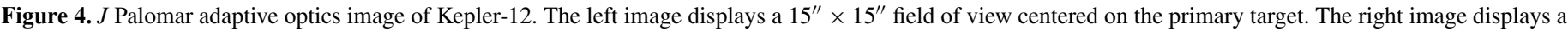

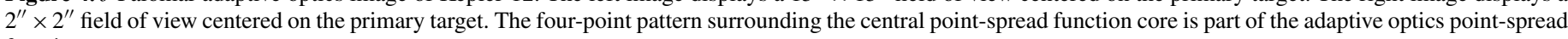
function.

(A color version of this figure is available in the online journal.) 


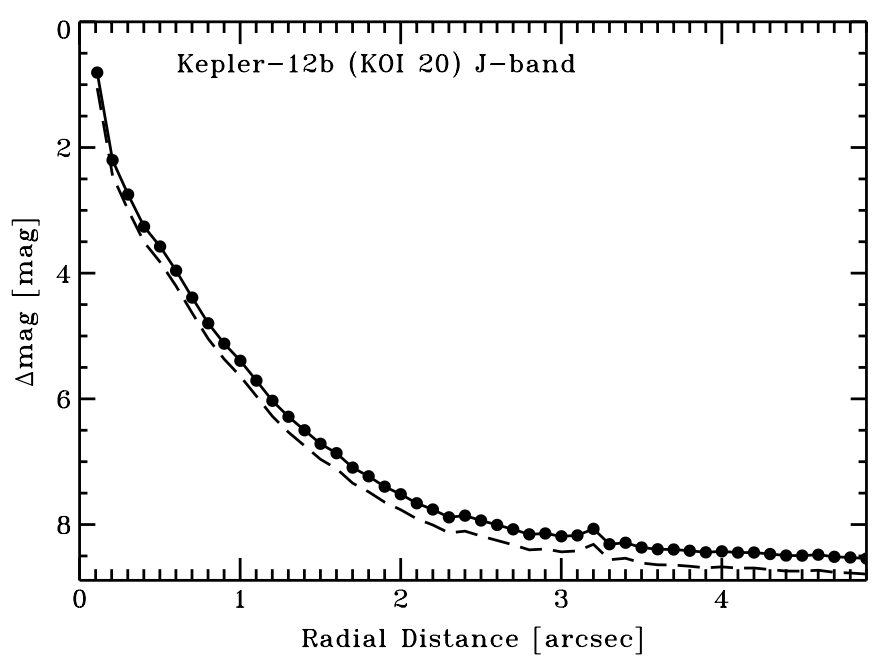

Figure 5. Palomar detection limits as a function of radial distance from the primary target, Kepler-12. The filled circles represent the $J$-band limits and each point represents a step in FWHM away from the primary target centroid peak. The dashed line underneath represents the $J$-band limits converted to Kepler magnitude limits if a star were to have a nominal $m_{\text {Kepler }}-J$ color. (For a magnitude-limited sample, the median $m_{\text {Kepler }}-J=1.28 \pm 0.52$.)

Table 1

Relative Radial Velocity and Bisector Span Variation Measurements of Kepler-12

\begin{tabular}{lrcrr}
\hline \hline BJD & $\begin{array}{c}\mathrm{RV} \\
\left(\mathrm{m} \mathrm{s}^{-1}\right)\end{array}$ & $\begin{array}{c}\sigma_{\mathrm{RV}} \\
\left(\mathrm{m} \mathrm{s}^{-1}\right)\end{array}$ & $\begin{array}{c}\mathrm{BS} \\
\left(\mathrm{m} \mathrm{s}^{-1}\right)\end{array}$ & $\begin{array}{c}\sigma_{\mathrm{BS}} \\
\left(\mathrm{m} \mathrm{s}^{-1}\right)\end{array}$ \\
\hline 2455014.91234 & -12.5 & 19.3 & 38.3 & 6.1 \\
2455016.79104 & 45.9 & 19.3 & 46.2 & 6.7 \\
2455017.10568 & 5.0 & 19.1 & 21.1 & 3.4 \\
2455019.11155 & 36.7 & 19.4 & 22.3 & 4.8 \\
2455027.05631 & -57.9 & 19.4 & 21.5 & 4.6 \\
2455041.99855 & -5.1 & 19.7 & 17.6 & 5.9 \\
2455042.79449 & 44.0 & 19.6 & 32.3 & 4.6 \\
2455073.83165 & 50.0 & 19.1 & -12.1 & 2.9 \\
2455075.83091 & -49.8 & 19.0 & -27.0 & 2.9 \\
2455080.87564 & -69.3 & 19.0 & -81.8 & 9.1 \\
2455084.86254 & -41.4 & 18.9 & 11.7 & 4.4 \\
2455134.82606 & 1.7 & 19.2 & -189.6 & 22.1 \\
2455437.78012 & 68.3 & 6.1 & 21.0 & 3.5 \\
2455439.75382 & -29.6 & 6.0 & 20.2 & 3.7 \\
2455759.86617 & -36.6 & 6.0 & 31.4 & 3.8 \\
2455761.82490 & 48.5 & 6.4 & 27.1 & 4.1 \\
\hline
\end{tabular}

visual magnitude of $V=13.8$, Kepler-12 was nonetheless observed with short exposure times of typically 10-30 minutes, yielding signal-to-noise ratios $(\mathrm{S} / \mathrm{Ns})$ near $\mathrm{S} / \mathrm{N}=30 \mathrm{pixel}^{-1}$ for most spectra. Such low-S/N taxes the Doppler code that was designed for much higher $\mathrm{S} / \mathrm{N}$, near 200. Thus, the wavelength scale and the instrumental profile were poorly determined, increasing the RV errors by unknown amounts. Moreover, all observations except the last four were made with a slit only 2.5 arcsec tall, preventing sky subtraction, which is now commonly applied to HIRES observations of faint Kepler stars taken after 2009 September. Moonlight certainly contaminated most of these spectra, as the moon was usually gibbous or full, adding systematic errors to the measured RVs. Thus, the RVs given here contain some poorly known errors that depend on the intensity and Doppler shift of the solar spectra relative to that of the star in the frame of the telescope. The velocities are given in Table 1.

Based on experience with other faint stars similarly observed, we expect true errors close to $18 \mathrm{~m} \mathrm{~s}^{-1}$ due to such effects, which

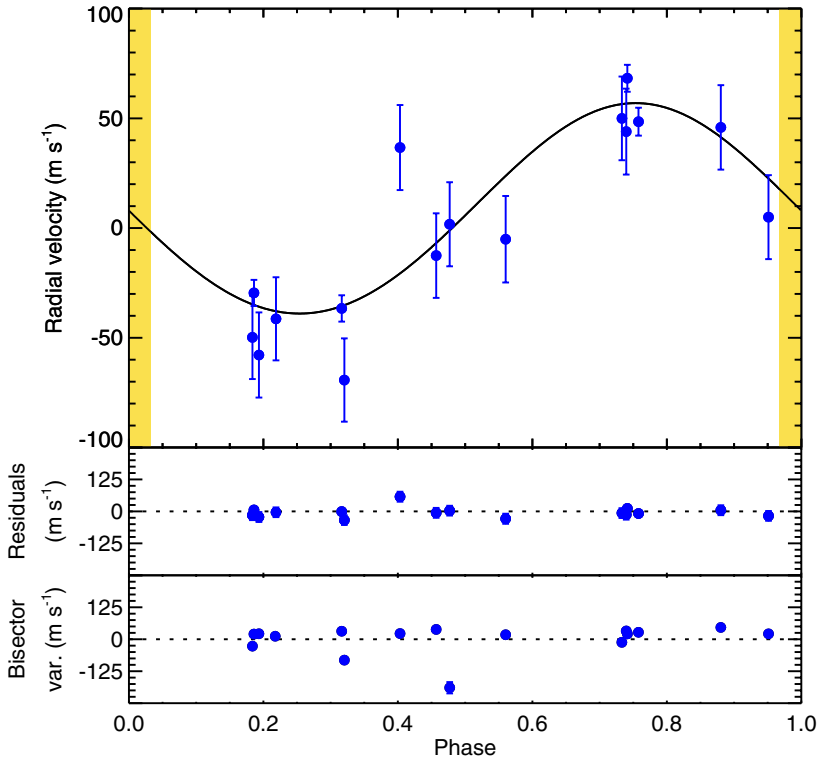

Figure 6. (a) Orbital solution for Kepler-12b. The observed radial velocities obtained with HIRES on the Keck telescope are plotted together with the velocity curve for a circular orbit with the period and time of transit fixed by the photometric ephemeris. The radial velocities have an arbitrary zero point. (b) Velocity residuals from the orbital solution. The rms of the velocity residuals is $24 \mathrm{~m} \mathrm{~s}^{-1}$. (c) Variation in the bisector spans for HIRES spectra. The zero point is arbitrary and the rms is $59 \mathrm{~m} \mathrm{~s}^{-1}$.

(A color version of this figure is available in the online journal.)

are here included in quadrature. Orbital analyses should include such uncertainties in applying weights to the RVs, albeit not Gaussian errors. The largest RV outlier to our orbital analysis is the fourth RV in Table 1 and appears at phase 0.4 in Figure 6. This measurement was made near morning twilight and may be more contaminated than the other measurements by sky spectrum. However, the measured mass of Kepler-12b is only modestly sensitive to these outliers; the mass of Kepler-12b increases by $7 \%$ when the largest RV outlier to a sinusoidal model is removed and the data are fit again.

The phased RV curve is shown in Figure 6. Since the orbital ephemeris from Kepler photometry was known a priori, observations were preferentially made at quadrature to allow the most robust determination of planetary mass with the fewest number of RV points. Observations were also made at additional phases to allow an initial estimate of orbital eccentricity. The RV observations can be further analyzed for bisector variations, which are shown in Figure 6(c). No variation that is in phase with the planetary orbit is found, which supports the planetary nature of the companion.

The RVs alone suggest a modest eccentricity, but a circular orbit certainly could not be eliminated with this data set. Since the long transit duration is the driver toward a large stellar radius, and hence a large planet radius, considerable care was taken to understand if an eccentric orbit around a smaller parent star could lead to the observed transit light curve (e.g., Barnes 2007). As shown in Sections 4 and 5, the timing and duration of the occultation put more robust constraints on eccentricity.

\section{DERIVATION OF STELLAR AND PLANETARY PARAMETERS}

\subsection{Kepler Photometry}

Our analysis is based on the Q0-Q7 data, representing nearly 1.5 years of data recorded in a quasi-continuous mode. Kepler 
data are in short-cadence (SC) and long-cadence (LC) time series, which are binnings per $58.84876 \mathrm{~s}$ and 29.4244 minutes, respectively, of the same CCD readouts. Eight LC (Jenkins et al. 2010b) and 16 SC (Gilliland et al. 2010) data sets are used as part of this study, representing 706,135 photometric data points and 516 effective days of observations, out of which 464 days have also been recorded in SC. We used the raw photometry for our purposes.

\subsection{Data Analysis}

For this global analysis, we used the implementation of the MCMC algorithm presented in Gillon et al. (2009, 2010). MCMC is a Bayesian inference method based on stochastic simulations that sample the posterior probability distributions of adjusted parameters for a given model. Our MCMC implementation uses the Metropolis-Hasting algorithm (e.g., Carlin $\&$ Lewis 2008) to perform this sampling. Our nominal model is based on a star and a transiting planet on a Keplerian orbit about their center of mass.

Our global analysis was performed using 213 light curves in total from Kepler. For the model fitting we use only the photometry near the transit events. Windows of width 0.8 days ( $18 \%$ of the orbit) surrounding transits were used to measure the local out-of-transit baseline, while minimizing the computation time. In the analysis $101 \mathrm{SC}$ time series were used for the transit photometry. The 1 minute cadence SC light curves yield excellent constraints on the transit parameters (e.g., Gilliland et al. 2010; Kipping 2010). Furthermore, 112 LC time series were employed for the occultation photometry. Input data to the MCMC also include the $16 \mathrm{RV}$ data points obtained from HIRES described in Section 3.2 and the four Spitzer 3.6 and $4.5 \mu \mathrm{m}$ occultation light curves described in Section 5.1.

The MCMC had the following set of jump parameters that are randomly perturbed at each step of the chains: the planet/star area ratio, the impact parameter $b^{\prime}=a \cos i / R_{\star}$, the transit duration from first to fourth contact, the time of inferior conjunction $T_{0}$ (HJD), the orbital period $P$ (assuming no transit timing variations), $K^{\prime}=K \sqrt{1-e^{2}} P^{1 / 3}$, where $K$ is the RV semi-amplitude, the occultation depth in Kepler and both Spitzer bandpasses, and the two parameters $\sqrt{e} \cos \omega$ and $\sqrt{e} \sin \omega$ (Anderson et al. 2011). A uniform prior distribution is assumed for all jump parameters. Kepler SC data allow a precise determination of the transit parameters and the stellar limbdarkening (LD) coefficients. We therefore assumed a quadratic law and used $c_{1}=2 u_{1}+u_{2}$ and $c_{2}=u_{1}-2 u_{2}$ as jump parameters, where $u_{1}$ and $u_{2}$ are the quadratic coefficients. Those linear combinations help in minimizing correlations on the uncertainties of $u_{1}$ and $u_{2}$ (Holman et al. 2006).

Three Markov chains of $10^{5}$ steps each were performed to derive the system parameters. Their good mixing and convergence were assessed using the Gelman-Rubin statistic (Gelman \& Rubin 1992).

At each step, the physical parameters are determined from the jump parameters above and the stellar mass. The transit and RV measurements together determine the planet orbit and allow for a geometrical measure of the mean density of the host star $\left(\bar{\rho}_{\star}\right)$. Using the MCMC chains, the probability distribution on $\overline{\rho_{\star}}$ was calculated, and together with the spectroscopically measured values and uncertainties of $T_{\text {eff }}$ and $[\mathrm{Fe} / \mathrm{H}]$, is used to determine consistent stellar parameters from Yonsei-Yale stellar evolution models (Demarque et al. 2004). The derived stellar $T_{\text {eff }}$ and $\bar{\rho}_{\star}$ parameters, compared to stellar evolution tracks, are shown in Figure 7 . The resulting normal distribution around

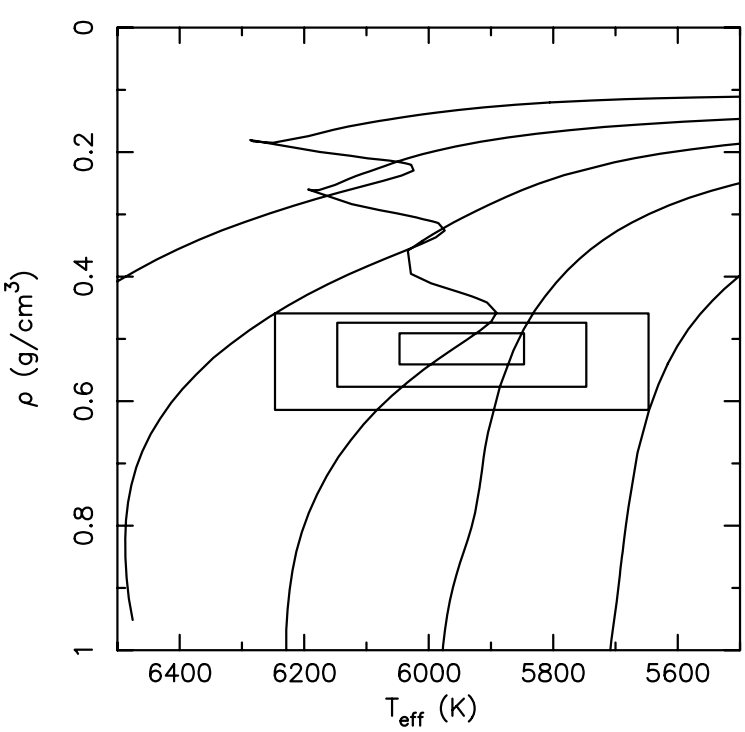

Figure 7. Five stellar evolution models from the Yonsei-Yale $\left(\mathrm{Y}^{2}\right)$ grids. From left to right the lines show $1.4,1.3,1.2,1.1$, and $1.0 M_{\odot}$ models for $Z=$ 0.0206 , which is appropriate given this parent star's metallicity, relative to the solar abundances used in the $\mathrm{Y}^{2}$ grids. The boxes show the $68.3 \%, 95.4 \%$, and $99.73 \%$ confidence intervals on the stellar $T_{\text {eff }}$ and $\bar{\rho}_{\star}$ as determined by spectroscopy and transit model fits. The "hook" in the evolution tracks for more massive stars with convective cores produces a non-uniform distribution of masses with the uncertainty boxes and can produce a degeneracy in the solution for the best-fit stellar parameters.

the stellar mass $(1.166 \pm 0.052) M_{\odot}$ was then used as a prior distribution in a new MCMC analysis, allowing the physical parameters of the system to be derived at each step of the chains.

\subsubsection{Model and Systematics}

The Kepler transit and occultation photometry are modeled with the Mandel \& Agol (2002) model, multiplied by a second-order polynomial accounting for stellar and instrumental variability. We added a quadratic function of the point-spread function (PSF) position to this baseline model for the Spitzer occultation light curves (see Section 5.1).

Baseline model coefficients are determined for each light curve with the singular value decomposition (SVD) method (Press et al. 1992) at each step of the MCMC. Correlated noise was accounted for following Winn et al. (2008) and Gillon et al. (2010) to ensure reliable error bars on the fitted parameters. For this purpose, we computed a scaling factor based on the standard deviation of the binned residuals for each light curve with different time bins. The error bars are then multiplied by this scaling factor. We obtained a mean scaling factor of 1.02 for all Kepler photometry, denoting a negligible contribution from correlated noise. The mean global Kepler photometric rms per 30 minute bin is 159 parts per million (ppm).

\subsection{Results}

We show in Table 2 the median values and the corresponding $68.3 \%$ probability interval of the posterior distribution function (PDF) for each parameter obtained from the MCMC. We present in Figure 8 the phase-folded transit photometry. We determine a planetary radius of $1.695_{-0.032}^{+0.028} R_{\mathrm{J}}$ and a mass of $0.431_{-0.040}^{+0.041} M_{\mathrm{J}}$ that produces a very low mean planetary density of $0.111_{-0.010}^{+0.011} \mathrm{~g} \mathrm{~cm}^{-3}$. 
Table 2

Kepler-12 System Parameters

\begin{tabular}{|c|c|}
\hline Parameters & Value \\
\hline \multicolumn{2}{|c|}{ Jump parameters } \\
\hline Planet/star area ratio $\left(R_{p} / R_{s}\right)^{2}$ & $0.013765_{-0.000020}^{+0.000020}$ \\
\hline$b^{\prime}=a \cos i / R_{\star}\left(R_{\star}\right)$ & $0.174_{-0.011}^{+0.011}$ \\
\hline Transit width (days) & $0.19573_{-0.00010}^{+0.00009}$ \\
\hline$T_{0}-2,450,000(\mathrm{HJD})$ & $5004.00835_{-0.00002}^{+0.00002}$ \\
\hline Orbital period $P$ (days) & $4.4379637_{-0.0000002}^{+0.0000002}$ \\
\hline $\mathrm{RV} K^{\prime}\left(\mathrm{m} \mathrm{s}^{-1}\right.$ day $\left.^{1 / 3}\right)$ & $79.2_{-7.0}^{+7.1}$ \\
\hline$\sqrt{e} \cos \omega$ & $-0.001_{-0.051}^{+0.054}$ \\
\hline$\sqrt{e} \sin \omega$ & $0.001_{-0.114}^{+0.097}$ \\
\hline$c_{1}=2 u_{1}+u_{2}$ & $1.009_{-0.005}^{+0.005}$ \\
\hline$c_{2}=u_{1}-2 u_{2}$ & $-0.182_{-0.014}^{+0.016}$ \\
\hline Occultation depth & $0.000031_{-0.000007}^{+0.000007}$ \\
\hline \multicolumn{2}{|c|}{ Deduced stellar parameters } \\
\hline$u_{1}$ & $0.367_{-0.003}^{+0.003}$ \\
\hline$u_{2}$ & $0.274_{-0.006}^{+0.006}$ \\
\hline Density $\rho_{\star}\left(\rho_{\odot}\right)$ & $0.354_{-0.008}^{+0.017}$ \\
\hline Surface gravity $\log g_{\star}(\mathrm{cgs})$ & $4.175_{-0.011}^{+0.015}$ \\
\hline Mass $M_{\star}\left(M_{\odot}\right)$ & $1.166_{-0.054}^{+0.051}$ \\
\hline Radius $R_{\star}\left(R_{\odot}\right)$ & $1.483_{-0.029}^{+0.025}$ \\
\hline Age (Gyr) & $4.0_{-0.4}^{+0.3}$ \\
\hline \multicolumn{2}{|c|}{ Observed stellar parameters } \\
\hline$T_{\text {eff }}$ & $5947 \pm 100$ \\
\hline$[\mathrm{Fe} / \mathrm{H}]$ & $0.07 \pm 0.04$ \\
\hline$V \sin i$ & $0.8 \pm 0.5 \mathrm{~km} \mathrm{~s}^{-1}$ \\
\hline \multicolumn{2}{|c|}{ Deduced planet parameters } \\
\hline $\mathrm{RV} K\left(\mathrm{~m} \mathrm{~s}^{-1}\right)$ & $48.2_{-4.3}^{+4.4}$ \\
\hline$b_{\text {transit }}\left(R_{\star}\right)$ & $0.174_{-0.011}^{+0.011}$ \\
\hline$b_{\text {occultation }}\left(R_{\star}\right)$ & $0.174_{-0.011}^{+0.011}$ \\
\hline$T_{\text {occultation }}-2,450,000(\mathrm{HJD})$ & $5010.666_{-0.003}^{+0.004}$ \\
\hline Orbital semi-major axis $a(\mathrm{AU})$ & $0.0556_{-0.0007}^{+0.0007}$ \\
\hline Orbital inclination $i(\mathrm{deg})$ & $88.76_{-0.08}^{+0.08}$ \\
\hline Orbital eccentricity $e$ & $<0.01(1 \sigma),<0.09(3 \sigma)$ \\
\hline Argument of periastron $\omega(\mathrm{deg})$ & $182_{-98}^{+97}$ \\
\hline Density $\rho_{P}\left(\mathrm{~g} \mathrm{~cm}^{-3}\right)$ & $0.111_{-0.009}^{+0.011}$ \\
\hline Surface gravity $\log g_{P}(\mathrm{cgs})$ & $2.57_{-0.04}^{+0.04}$ \\
\hline Mass $M_{P}\left(M_{\mathrm{Jup}}\right)$ & $0.431_{-0.040}^{+0.041}$ \\
\hline Radius $R_{P}\left(R_{\mathrm{Jup}}\right)$ & $1.695_{-0.032}^{+0.028}$ \\
\hline
\end{tabular}

We measure occultation depths of $0.099 \% \pm 0.028 \%$ and $0.116 \% \pm 0.034 \%$ in Spitzer IRAC 3.6 and $4.5 \mu \mathrm{m}$ channels, respectively, consistent at the $1 \sigma$ level with the specific analysis present in Section 5.1. The LD quadratic coefficients derived from the MCMC are $u_{1}=0.375 \pm 0.004$ and $u_{2}=0.250 \pm 0.008$. These are in good agreement with the theoretical coefficients obtained from the Claret \& Bloemen (2011) tables of $u_{1}=0.366$ and $u_{2}=0.275$.

We finally determine an occultation depth of $31 \pm 8 \mathrm{ppm}$ in the Kepler bandpass, which corresponds to a geometric albedo $A_{g}=0.14 \pm 0.04$. The geometric albedo is wavelength dependent and measures the ratio of the planet flux at zero phase angle to the flux from a Lambert disk at the same distance and the same cross-sectional area as the planet (see, e.g., Marley
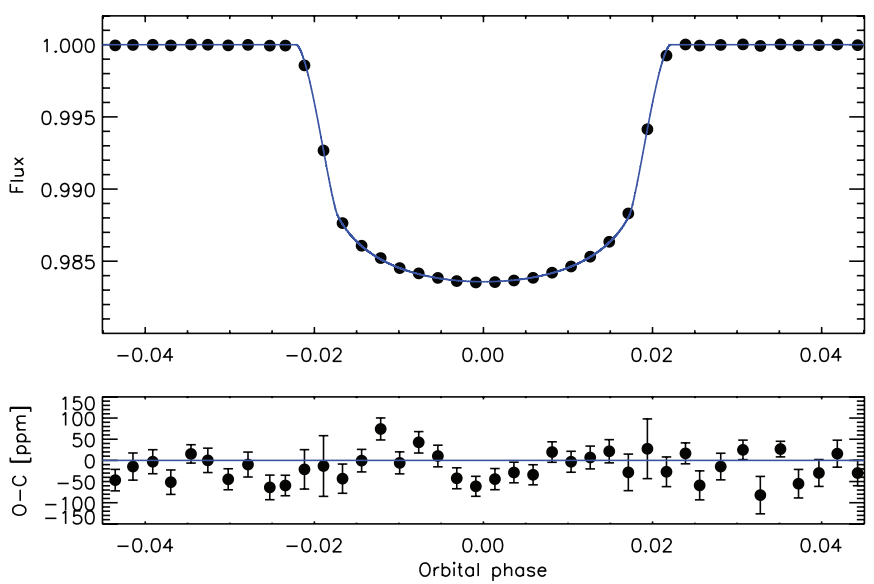

Figure 8. Top: Kepler-12b phase-folded transit light curve with best-fit model superimposed. The data are binned in 15 minute intervals. Error bars are smaller than the plotted data points. Bottom: residuals are displayed in parts per million (ppm) scale.

(A color version of this figure is available in the online journal.)
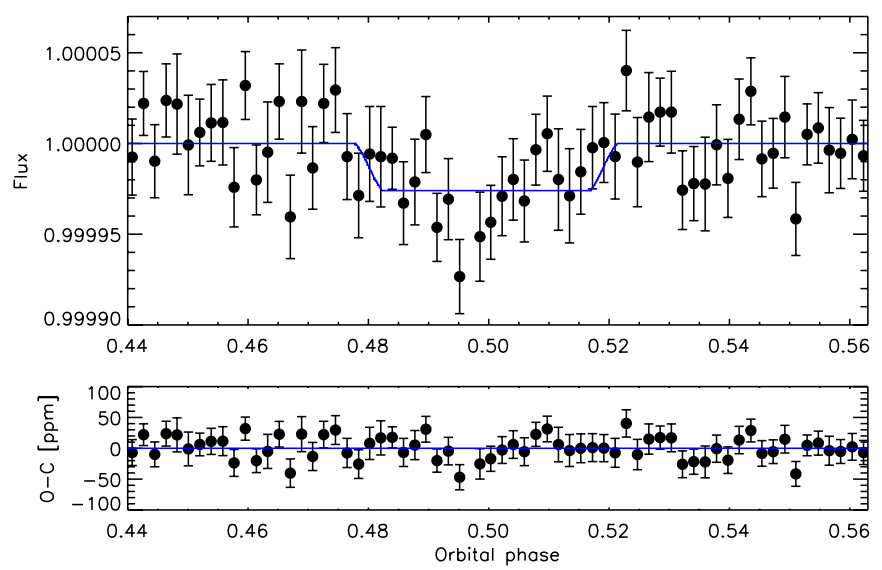

Figure 9. Top: Kepler-12 b phase-folded occultation light curve with best-fit model superimposed. The Kepler data are binned in 15 minute increments. Bottom: residuals are displayed in parts per million (ppm) scale.

(A color version of this figure is available in the online journal.)

et al. 1999; Sudarsky et al. 2000):

$$
\frac{F_{p}}{F_{\star}}=A_{g}\left(\frac{R_{p}}{a}\right)^{2},
$$

where $F_{p} / F_{\star}$ is the occultation depth, $a$ the orbital semi-major axis, and $R_{p}$ the planetary radius.

The corresponding phase-folded occultation light curve is shown in Figure 9. The combination of Spitzer and Kepler occultations leads to a $1 \sigma$ orbital eccentricity signal of $e<0.01$, while the $3 \sigma$ limit is $e<0.09$. We show $e \sin \omega$ versus $e \cos \omega$ from successful MCMC trials in Figure 10. The small allowed eccentricity removes most solutions that allow fits to the long transit duration with smaller stellar (and planetary) radii. There are two paths toward a more robust constraint on $e$. One would come from many additional RV points. An easier path would be additional quarters of Kepler data, which would yield a better determination of the occultation duration, which constrains $e \sin \omega$. All system parameters are collected in Table 2 . 
Table 3

Warm-Spitzer Observations

\begin{tabular}{|c|c|c|c|c|c|c|c|}
\hline Visit & $\mathrm{AOR}$ & Wavelength & Obs. Date (UT) & Select. Points & Depth $(\%)$ & Weighted. Avg. Depth & $T_{\text {bright }}$ \\
\hline 1 & 40251392 & 3.6 & 2010 Sep 6 & 1233 & $0.141_{-0.021}^{+0.026}$ & $\cdots$ & $\ldots$ \\
\hline 3 & 40250880 & 3.6 & 2010 Dec 26 & 1151 & $0.130_{-0.032}^{+0.026}$ & $0.137 \pm 0.020 \%$ & $1585 \pm 170 \mathrm{~K}$ \\
\hline 4 & 40250624 & 4.5 & 2011 Jan 8 & 1212 & $0.129_{-0.061}^{+0.039}$ & $0.116 \pm 0.031 \%$ & $1420 \pm 200 \mathrm{~K}$ \\
\hline
\end{tabular}

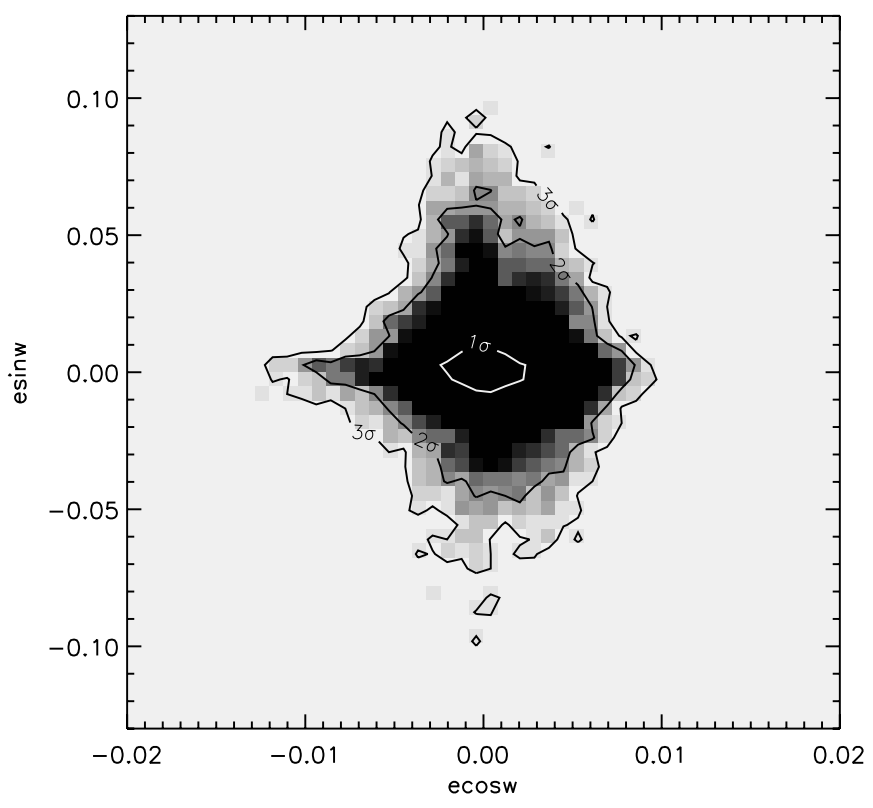

Figure 10. Density function of the two-dimensional $e \sin \omega / e \cos \omega$ successful MCMC trials (density increases from white to black). Note the different scales for the $x$ - and $y$-axes. The $1 \sigma, 2 \sigma$, and $3 \sigma$ confidence domains are superimposed. The weak constraint from the occultation duration leads to a significant scatter in $e \sin \omega$. As $e \cos \omega$ is well constrained from the occultation timing obtained by the Kepler and Spitzer photometry, this yields orbital eccentricities as high as $\sim 0.09$ ( $3 \sigma$ upper limit) provided the argument of the periastron is close to $90^{\circ}$ or $270^{\circ}$. In this case, the eccentricity vector points toward or away of the observer, allowing a wide range of $e \sin \omega$ values while leaving $e \cos \omega$ almost unchanged.

\section{ATMOSPHERIC CHARACTERIZATION AT SECONDARY ECLIPSE}

As part of Spitzer program 60028 (PI: D. Charbonneau) a number of Kepler-detected giant planets were observed in order to characterize the planets' thermal emission at 3.6 and $4.5 \mu \mathrm{m}$ during the Warm Spitzer extended mission. The inherent faintness of the planetary targets mean some stars must be observed more than once for adequate signal to noise to enable meaningful atmospheric characterization.

In addition to the measurement of the depth of the occultation (or secondary eclipse), which yields a measurement of the planetary brightness temperature, the timing and duration of the occultation constrains $e$, as described above. The timing of the transit constrains $e \cos \omega$ where $\omega$ is the longitude of periapse. The duration of the transit constrains $e \sin \omega$. The former is generally easier to measure accurately than the latter.

\subsection{Warm-Spitzer Detections}

Kepler-12 was observed during four occultations between 2010 August and 2011 January with Warm-Spitzer/IRAC (Werner et al. 2004; Fazio et al. 2004) at 3.6 and $4.5 \mu \mathrm{m}$. Two occultations were gathered per bandpass and each visit lasted approximately $11 \mathrm{hr}$. The data were obtained in full-frame mode $(256 \times 256$ pixels) with an exposure time of $30.0 \mathrm{~s}$ per image which yielded 1321 images per visit. The set of observations is shown in Table 3.

The method we used to produce photometric time series from the images is described in Désert et al. (2011a). It consists of finding the centroid position of the stellar PSF and performing aperture photometry using a circular aperture on individual exposures. The images used are the Basic Calibrated Data (BCD) delivered by the Spitzer archive. These files are corrected for dark current, flat fielding, detector nonlinearity, and converted into flux units. We convert the pixel intensities to electrons using the information on detector gain and exposure time provided in the FITS headers. This facilitates the evaluation of the photometric errors. We extract the UTC-based Julian date for each image from the FITS header and correct to mid-exposure. We then correct for transient pixels in each individual image using a 20-point sliding median filter of the pixel intensity versus time. To do so, we compare each pixel's intensity to the median of the 10 preceding and 10 following exposures at the same pixel position and we replace outliers greater than $3 \sigma$ with its median value. The fraction of pixels we correct varies between $0.15 \%$ and $0.22 \%$ depending on the visit. The centroid position of the stellar PSF is determined using DAOPHOT-type Photometry Procedures, GCNTRD, from the IDL Astronomy Library. ${ }^{22}$ We use the APER routine to perform aperture photometry with a circular aperture of variable radius, using radii of $1.5-8$ pixels in 0.5 steps. The propagated uncertainties are derived as a function of the aperture radius; we adopt the one that provides the smallest errors. We find that the transit depths and errors vary only weakly with the aperture radius for all the light curves analyzed in this project. The optimal apertures are found to have radii of 2.5 pixels.

We estimate the background by fitting a Gaussian to the central region of the histogram of counts from the full array. The center of the Gaussian fit is adopted as the residual background intensity. As already seen in previous Warm-Spitzer observations (Deming et al. 2011; Beerer et al. 2011), we find that the background varies by $20 \%$ between three distinct levels from image to image and displays a ramp-like behavior as a function of time. The contribution of the background to the total flux from the stars is low for both observations, from $0.07 \%$ to $1.2 \%$ depending on the images. Therefore, photometric errors are not dominated by fluctuations in the background. We used a sliding median filter to select and trim outliers in flux and positions greater than $4 \sigma$. This process removes between $0.9 \%$ and $2.8 \%$ of the data, depending on the visit. We also discarded the first half-hour of observations, which are affected by a significant telescope jitter before stabilization. The final number of photometric measurements used is presented in Table 3. The raw time series are presented in the top panels of Figure 11.

\footnotetext{
22 http://idlastro.gsfc.nasa.gov/homepage.html
} 

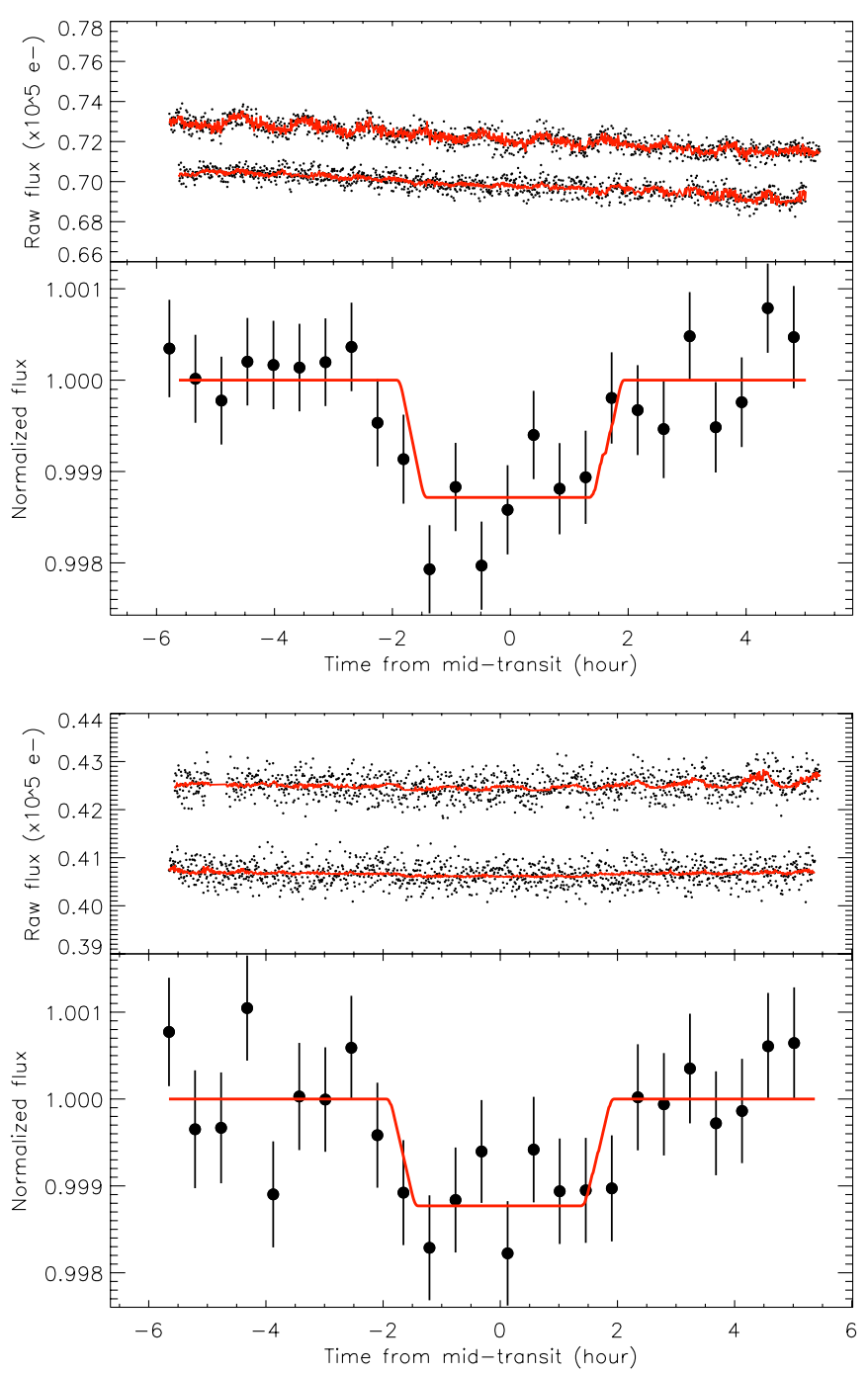

Figure 11. Spitzer occultation light curves of Kepler-12b observed in the IRAC bandpass at 3.6 (top) and $4.5 \mu \mathrm{m}$ (bottom). Top panels: raw and unbinned light curves. The red solid lines correspond to the best-fit models which include the time and position instrumental decorrelations as well as the model for the planetary transit (see details in Section 5.1). Bottom panels: corrected and normalized occultation light curve with the best-fit model (in red). The data are binned in 25 minute intervals ( 50 points).

(A color version of this figure is available in the online journal.)

We find that the point-to-point scatter in the photometry gives a typical $\mathrm{S} / \mathrm{N}$ of 260 and 200 per image at 3.6 and $4.5 \mu \mathrm{m}$, respectively. These correspond to $85 \%$ of the theoretical signal to noise. Therefore, the noise is dominated by Poisson photon noise. We used a transit light curve model multiplied by instrumental decorrelation functions to measure the occultation parameters and their uncertainties from the Spitzer data as described in Désert et al. (2011b). We compute the transit light curves with the IDL transit routine OCCULTSMALL from Mandel $\&$ Agol (2002). In the present case, this function depends on one parameter: the occultation depth $d$. The planet-to-star radius ratio $R_{p} / R_{\star}$, the orbital semi-major axis to stellar radius ratio (system scale) $a / R_{\star}$, the mid-occultation time $T_{c}$, and the impact parameter $b$ are set fixed to the values derived from the Kepler light curves.

The Spitzer/IRAC photometry is known to be systematically affected by the so-called pixel-phase effect (see, e.g., Charbonneau et al. 2005; Knutson et al. 2008). This effect is seen as oscillations in the measured fluxes with a period of ap- proximately 70 minutes (period of the telescope pointing jitter) and an amplitude of approximately $2 \%$ peak-to-peak. We decorrelated our signal in each channel using a linear function of time for the baseline (two parameters) and a quadratic function of the PSF position (four parameters) to correct the data for each channel. We performed a simultaneous Levenberg-Marquardt least-squares fit (Markwardt 2009) to the data to determine the occultation depth and instrumental model parameters (seven in total). The errors on each photometric point were assumed to be identical and were set to the rms of the residuals of the initial best-fit model. To obtain an estimate of the correlated and systematic errors (Pont et al. 2006) in our measurements, we use the residual permutation bootstrap or "Prayer Bead" method as described in Désert et al. (2009). In this method, the residuals of the initial fit are shifted systematically and sequentially by one frame, and then added to the transit light curve model before fitting again. We allow asymmetric error bars spanning 34\% of the points above and below the median of the distributions to derive the $1 \sigma$ uncertainties for each parameter as described in Désert et al. (2011a).

We measure the occultation depths in each bandpass and for each individual visit. The values we measure for the depths are all in agreement at the $1 \sigma$ level. Furthermore the weighted mean averages per bandpass of the transit depths are consistent with the depths derived by the global Monte Carlo analysis.

\subsection{Joint Constraints on the Atmosphere}

To model the planet's atmosphere we use a one-dimensional plane-parallel atmosphere code that has been widely used for solar system planets, exoplanets, and brown dwarfs over the past two decades. The optical and thermal infrared radiative transfer solvers are described in detail in Toon et al. (1989). Past applications of the model include Titan (McKay et al. 1989), Uranus (Marley \& McKay 1999), gas giant exoplanets (Fortney et al. 2006, 2008; Fortney \& Marley 2007), and brown dwarfs (Marley et al. 1996; Burrows et al. 1997; Marley et al. 2002; Saumon \& Marley 2008). We use the correlated- $k$ method for opacity tabulation (Goody et al. 1989). Our extensive opacity database is described in Freedman et al. (2008). We make use of tabulations of chemical mixing ratios from equilibrium chemistry calculations of K. Lodders and collaborators (Lodders 1999; Lodders \& Fegley 2002, 2006). We use the protosolar abundances of Lodders (2003). Since the first detection of thermal flux from hot Jupiters (Charbonneau et al. 2005; Deming et al. 2005) we have used the code extensively to model strongly irradiated planet atmospheres and have compared model spectra to observations (e.g., Fortney et al. 2005; Knutson et al. 2009; Deming et al. 2011; Désert et al. 2011a).

Planet Kepler-12b intercepts an incident flux of $1.1 \times$ $10^{9} \mathrm{erg} \mathrm{s}^{-1} \mathrm{~cm}^{-2}$, a value just larger than the suggested $\mathrm{pM} / \mathrm{pL}$ class incident flux boundary proposed by Fortney et al. (2008). It was suggested that planets warmer than this boundary (pM) would harbor dayside temperature inversions, while those cooler than this boundary would not have inversions. It is therefore important to understand the temperature structure of the planet. For Kepler-12b we show three models in Figure 12, for which we plot the planet-to-star flux ratio and dayside $P-T$ profiles. In red and blue are "dayside average" models with incident flux redistributed over the dayside only. In green is a model where the incident flux is cut in half to simulate efficient redistribution of energy to the night side (see, e.g., Fortney \& Marley 2007). The model in red has a temperature inversion due to absorption of incident flux by $\mathrm{TiO}$ and VO vapor (e.g., Hubeny et al. 2003; 


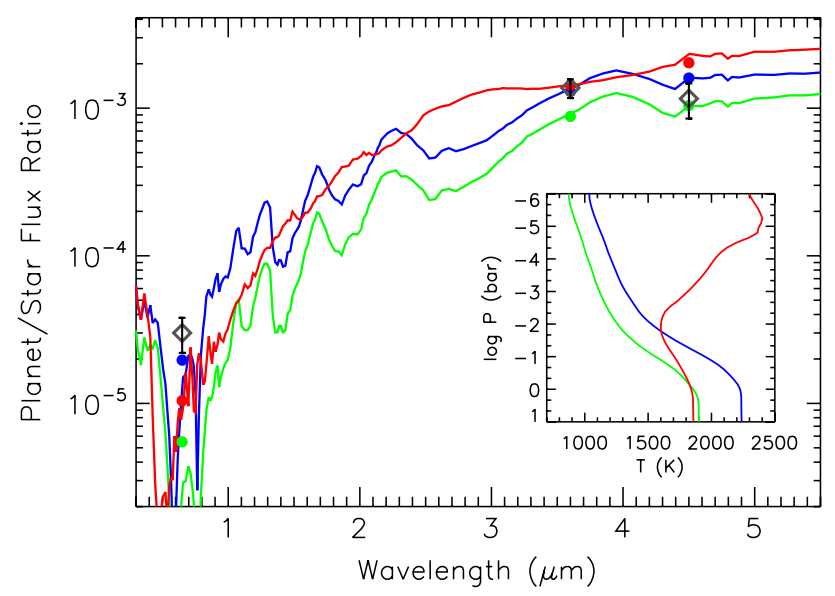

Figure 12. Main panel: planet-to-star flux ratios observed by Kepler and Spitzer, shown in gray. The flux ratios from three planetary models are shown for comparison. There is preference toward models that have no temperature inversion (blue and green). Model ratios integrated over the appropriate bandpasses are shown as filled circles. The Kepler occultation point strongly argues for inefficient redistribution of flux or an additional scattering component at optical wavelengths. Inset panel: atmospheric pressure-temperature profiles for the three models.

(A color version of this figure is available in the online journal.)

Fortney et al. 2008), while the blue and green models lack inversions, as $\mathrm{TiO} / \mathrm{VO}$ vapor is removed from the opacities. The Kepler occultation depth is shown at $0.65 \mu \mathrm{m}$ (diamond), while the Spitzer detections are shown as diamonds at 3.6 and $4.5 \mu \mathrm{m}$. Model band averages at these wavelengths are shown as solid circles.

The relatively flat ratio of the 3.6/4.5 diamond points generally points to a very weak or no inversion (Knutson et al. 2010). Looking to the optical, the green model is dramatically too dim, while the blue model nearly reaches the $1 \sigma$ error bar. Looking at the infrared, the blue point is at the $1 \sigma 4.5 \mu \mathrm{m}$ error bar as well. The inverted model (red) has approximately the same $T_{\text {eff }}(\sim 1700 \mathrm{~K})$ as the blue model, but higher fluxes in the mid-infrared and lower fluxes in the near-infrared and optical. The Spitzer data alone do not give us strong leverage on the temperature structure. Any cooler model with an inversion (not plotted) would yield a better fit to Spitzer and a worse fit to Kepler. Within the selection of models, the brightness of the Kepler point argues for the no-inversion model. The flux in the Kepler band from the blue model is $60 \%$ scattered light, $40 \%$ thermal emission.

Our tentative conclusion is that the blue (no inversion, inefficient temperature homogenization onto the night side) model is preferred. However, given our ignorance of the optical opacity in these atmospheres, this conclusion is tentative. The relatively deep occultation in the Kepler band argues for an additional contribution at optical wavelengths that is not captured in the model. One possibility is that stellar flux has photoionized $\mathrm{Na}$ and K gases (Fortney et al. 2003), which are thought to be strong absorbers of stellar light (and therefore diminish scattered light) in hot Jupiter atmospheres. Another possibility is a population of small grains, such as silicates, which could scatter some stellar flux (Marley et al. 1999; Seager et al. 2000; Sudarsky et al. 2000). Such clouds are prominent in L-dwarf atmospheres (e.g., Ackerman \& Marley 2001).

\section{DISCUSSION}

A great number of explanations have been put forward to explain the inflated radii of the close-in giant planets.
They generally fall into several broad classes and are recently reviewed in Fortney \& Nettelmann (2010) and Baraffe et al. (2010). Some argue for a delayed contraction due to slowed energy transport in the atmosphere (Burrows et al. 2007) or the deep interior (Chabrier \& Baraffe 2007). Others suggest a variety of atmospheric effects (Showman \& Guillot 2002; Guillot \& Showman 2002; Batygin \& Stevenson 2010; Arras \& Socrates 2010; Youdin \& Mitchell 2010) that lead to energy dissipation into the interior. Still others suggest tidal dissipation in the interior due to eccentricity damping (Bodenheimer et al. 2000; Jackson et al. 2008; Miller et al. 2009; Ibgui \& Burrows 2009).

For Kepler-12b, we do not find evidence for transit timing variations (Ford et al. 2011). The rms scatter of transit times about a linear ephemeris is less than one minute and only $17 \%$ larger than the average of the formal timing uncertainties. This rules out the presence of massive non-transiting planets very near by or in the outer 1:2 mean motion resonance. In principle, a more distant non-resonant planet is possible, but hot Jupiters rarely have a second massive planet close to the star (Wright et al. 2009, 2011; Latham et al. 2011). Thus, it is very unlikely that the inflated radius is due to eccentricity damping.

Clarity on a radius-inflation mechanism has not been achieved, but Figure 1 appears to argue for an explanation based on the planet temperature or irradiation level of the atmosphere (rather than merely on orbital separation), as has been shown by other authors (Kovács et al. 2010; Laughlin et al. 2011; Batygin et al. 2011).

If the inflation mechanism can be thought of as an energy source that is added to the planet's deep convective interior, we can readily compare the energy input needed to sustain the radius of Kepler-12b, compared to other planets. This is actually more physically motivated than the more commonly discussed "radius anomaly," since the power needed to inflate the radius by a given amount, $\Delta R$, is a very strong function of mass. In particular, Figure 6 in Miller et al. (2009) allows for a comparison of input power as a function of planet mass for 4.5 Gyr old model planets with $10 M_{\oplus}$ cores at $0.05 \mathrm{AU}$ from the Sun. For instance, inflating a $0.2 M_{\mathrm{J}}$ planet by $0.2 R_{\mathrm{J}}$ over its expected radius value takes $1 \times 10^{24} \mathrm{erg} \mathrm{s}^{-1}$, while for a $2 M_{\mathrm{J}}$ planet it is $2 \times 10^{27} \mathrm{erg}^{-1}$, a factor of 2000 difference in power for a factor of 10 in mass. This is the reason why Batygin et al. (2011) can easily expand Saturn-mass planets to the point of disruption via Ohmic dissipation - a small amount of energy goes a long way toward inflating the radii of low-mass planets.

In understanding the structure of Kepler-12b, we can use the models described in Miller et al. (2009), which are adapted from Fortney et al. (2007). In particular, Table 1 in Miller et al. (2009) includes the current internal power necessary to explain the radius of several inflated planets. Planets HD 209458b (Henry et al. 2000; Charbonneau et al. 2000) and TrES-4b (Mandushev et al. 2007) are interesting points of comparison. HD 209458b and Kepler-12b have similar incident fluxes, while TrES-4b and Kepler-12b have similar inflated radii.

Since Kepler-12b and HD 209458b have comparable incident stellar fluxes (that of Kepler-12b is $14 \%$ larger), one could easily assume that they have similar interior energy sources (e.g., Guillot \& Showman 2002). For HD 209458b, with core masses of 0,10 , and $30 M_{\oplus}$, incident powers of $1.5 \times 10^{26}, 3.8 \times 10^{26}$, and $1.6 \times 10^{27} \mathrm{erg} \mathrm{s}^{-1}$, are required (Miller et al. 2009). Using the planetary parameters of Kepler- $12 \mathrm{~b}$ with cores of 0,10 , and $30 M_{\oplus}$, the required powers are substantially larger. The enhancement is generally a factor of three larger, with values of 


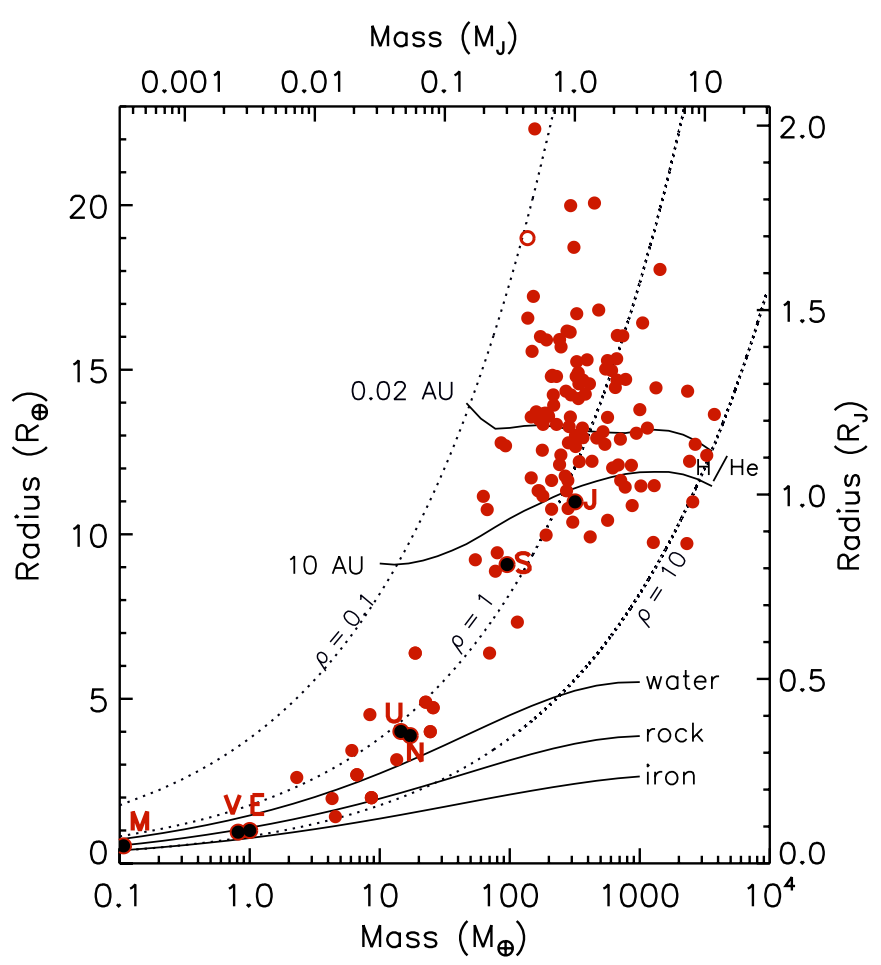

Figure 13. Mass vs. radius for planets with "well-defined parameters," as taken from http://www.inscience.ch/transits/, but also including the Kepler-11 system (Lissauer et al. 2011). Kepler-12b is shown as an open circle, the second lowest density planet discovered. Models (solid black curves) are taken from Fortney et al. (2007). The two upper curves are for pure $\mathrm{H}-\mathrm{He}$ planets, at $4.5 \mathrm{Gyr}$, at 0.02 and $10 \mathrm{AU}$ from the Sun.

(A color version of this figure is available in the online journal.)

$4.4 \times 10^{26}, 1.1 \times 10^{27}$, and $4.2 \times 10^{27} \mathrm{erg} \mathrm{s}^{-1}$, respectively. This could point to more than one radius-inflation mechanism being at play in this planet, as has recently been strongly suggested for the massive transiting planet CoRoT-2b (Guillot \& Havel 2011).

The difference between Kepler-12b and HD 209458b can be remedied, however, if the planets have different heavyelement masses. In particular, both planets would require power levels of $\sim 1.6 \times 10^{27} \mathrm{erg} \mathrm{s}^{-1}$ if Kepler-12b possesses only $\sim 15 M_{\oplus}$ of heavy elements, while HD $209458 \mathrm{~b}$ possesses $30 M_{\oplus}$. The Kepler-12b parent star has an $[\mathrm{Fe} / \mathrm{H}]=+0.07$, while for HD 209458 it is +0.02 . As recently shown by Miller $\&$ Fortney (2011) for the colder non-inflated planets, for parent stars with similar stellar metallicities, a spread from 10-30 $M_{\oplus}$ is reasonable. Therefore, it appears that the wide disparity in radii between these two well-studied planets could alternatively be due to the differences in interior heavy-element masses. Large diversities in heavy-element abundances are clearly needed to explain plots like Figure 13, where planets of similar masses can have dramatically different radii.

For comparison, TrES-4b at $0.93 M_{\mathrm{J}}$ and $1.78 R_{\mathrm{J}}$ is nearly twice as massive as Kepler-12b, but intercepts 2.1 times higher incident flux. The inflation powers at 0,10 , and $30 M_{\oplus}$ range from 1.0 to $3.4 \times 10^{28}, 8-20$ times larger than for Kepler-12b, at the same heavy-element masses. Clearly the required energy difference between the two models does not scale simply with the incident flux. As discussed in Miller \& Fortney (2011) as the population of cool $\left(T_{\mathrm{eq}}<1000 \mathrm{~K}\right)$ non-inflated planets grows, the heavy-element mass of extrasolar gas giants can become better understood as a function of planet mass and stellar metallicity, which will allow for more robust constraints on the heavy-element masses of the inflated planets. This will in turn allow for better estimates of the magnitude of the additional interior energy source within these planets. While Kepler-12b does not quite fit the general trend that the highest irradiation planets are the largest, this trend argues for an explanation that for the most part scales with atmospheric temperature. A more detailed computational understanding of how the visible atmosphere, deep atmosphere, and convective interior interact and feedback on each other is now clearly needed.

\section{CONCLUSIONS}

We report the discovery of planet Kepler-12b from transit observations by Kepler. The planet has an unusually inflated radius and low bulk density. At its incident flux level, the large radius of the planet makes it somewhat of an outlier compared to the general empirical trend that the most inflated planets intercept the highest incident fluxes. This may require the planet to have an usually low-mass fraction of heavy elements within its interior, or that more than one radius-inflation mechanism is at work in its interior.

The atmosphere of the planet is probed via detections of the occultations in the Kepler and Warm-Spitzer bandpasses. Given the faintness of the parent star, characterization was difficult, but all detections were made at a level of at least 3.5 $\sigma$. A model comparison to the data yields a best-fit model that lacks a dayside temperature inversion, given the relatively flat $3.6 / 4.5 \mu \mathrm{m}$ ratio of the planet-to-star flux ratios, along with the relatively large occultation depth in the Kepler band. Additional Kepler data will yield more robust constraints on the planet's geometric albedo, orbital eccentricity, and perhaps phase curve information.

J.J.F. acknowledges the support of the Kepler Participating Scientist's program via NASA grant NNX09AC22G. Kepler was competitively selected as the tenth Discovery mission. Funding for the Kepler mission is provided by NASA's Science Mission Directorate. We thank the many people who gave so generously of their time to make the Kepler mission a success. We thank Carly Chubak for cross-correlation analyses of the Keck spectra for companions. This work incorporates observations made with the Spitzer Space Telescope, which is operated by the Jet Propulsion Laboratory, California Institute of Technology under a contract with NASA. Support for this work was provided by NASA through an award issued by JPL/Caltech. Finally, the authors extend special thanks to those of Hawaiian ancestry on whose sacred mountain of Mauna Kea we are privileged to be guests. Without their generous hospitality, the Keck observations presented herein would not have been possible.

\section{REFERENCES}

Ackerman, A. S., \& Marley, M. S. 2001, ApJ, 556, 872

Anderson, D. R., Collier Cameron, A., Hellier, C., et al. 2011, ApJ, 726, L19 Anderson, D. R., Hellier, C., Gillon, M., et al. 2010, ApJ, 709, 159 Arras, P., \& Socrates, A. 2010, ApJ, 714, 1

Baraffe, I., Chabrier, G., \& Barman, T. 2008, A\&A, 482, 315 Baraffe, I., Chabrier, G., \& Barman, T. 2010, Rep. Prog. Phys., 73, 016901 Barnes, J. W. 2007, PASP, 119, 986

Batygin, K., \& Stevenson, D. J. 2010, ApJ, 714, L238

Batygin, K., Stevenson, D. J., \& Bodenheimer, P. H. 2011, ApJ, 738, 1 Beerer, I. M., Knutson, H. A., Burrows, A., et al. 2011, ApJ, 727, 23 Bodenheimer, P., Hubickyj, O., \& Lissauer, J. J. 2000, Icarus, 143, 2 Bodenheimer, P., Laughlin, G., \& Lin, D. N. C. 2003, ApJ, 592, 555 Borucki, W. J., Koch, D., Basri, G., et al. 2010, Science, 327, 977 
Borucki, W. J., Koch, D. G., Basri, G., et al. 2011, ApJ, 728, 117

Burrows, A., Hubeny, I., Budaj, J., \& Hubbard, W. B. 2007, ApJ, 661, 502

Burrows, A., Ibgui, L., \& Hubeny, I. 2008, ApJ, 682, 1277

Burrows, A., Marley, M., Hubbard, W. B., et al. 1997, ApJ, 491, 856

Carlin, B. P., \& Lewis, B. P. 2008, Bayesian Methods for Data Analysis (3rd ed.; London: Chapman \& Hall)

Chabrier, G., \& Baraffe, I. 2007, ApJ, 661, L81

Charbonneau, D., Allen, L. E., Megeath, S. T., et al. 2005, ApJ, 626, 523

Charbonneau, D., Brown, T. M., Burrows, A., \& Laughlin, G. 2007, in Protostars and Planets V, ed. B. Reipurth, D. Jewitt, \& K. Keil (Tucson, AZ: Univ. Arizona Press), 701

Charbonneau, D., Brown, T. M., Latham, D. W., \& Mayor, M. 2000, ApJ, 529, L45

Claret, A., \& Bloemen, S. 2011, A\&A, 529, A75

Cowan, N. B., \& Agol, E. 2011, ApJ, 729, 54

Demarque, P., Woo, J.-H., Kim, Y.-C., \& Yi, S. K. 2004, ApJS, 155, 667

Deming, D., Knutson, H., Agol, E., et al. 2011, ApJ, 726, 95

Deming, D., Seager, S., Richardson, L. J., \& Harrington, J. 2005, Nature, 434, 740

Désert, J.-M., Charbonneau, D., Fortney, J. J., et al. 2011a, ApJ, submitted (arXiv:1102.0555)

Désert, J.-M., Lecavelier des Etangs, A., Hébrard, G., et al. 2009, ApJ, 699, 478

Désert, J., Sing, D., Vidal-Madjar, A., et al. 2011b, A\&A, 526, A12

Fazio, G. G., Hora, J. L., Allen, L. E., et al. 2004, ApJS, 154, 10

Ford, E. B., Rowe, J. F., Fabrycky, D. C., et al. 2011, ApJS, 197, 2

Fortney, J. J., Lodders, K., Marley, M. S., \& Freedman, R. S. 2008, ApJ, 678, 1419

Fortney, J. J., \& Marley, M. S. 2007, ApJ, 666, L45

Fortney, J. J., Marley, M. S., \& Barnes, J. W. 2007, ApJ, 659, 1661

Fortney, J. J., Marley, M. S., Lodders, K., Saumon, D., \& Freedman, R. 2005, ApJ, 627, L69

Fortney, J. J., \& Nettelmann, N. 2010, Space Sci. Rev., 152, 423

Fortney, J. J., Saumon, D., Marley, M. S., Lodders, K., \& Freedman, R. S. 2006, ApJ, 642, 495

Fortney, J. J., Sudarsky, D., Hubeny, I., et al. 2003, ApJ, 589, 615

Freedman, R. S., Marley, M. S., \& Lodders, K. 2008, ApJS, 174, 504

Gelman, A., \& Rubin, D. 1992, Stat. Sci., 7, 457

Gilliland, R. L., Jenkins, J. M., Borucki, W. J., et al. 2010, ApJ, 713, L160

Gillon, M., Demory, B.-O., Triaud, A. H. M. J., et al. 2009, A\&A, 506, 359

Gillon, M., Lanotte, A. A., Barman, T., et al. 2010, A\&A, 511, A3

Goody, R., West, R., Chen, L., \& Crisp, D. 1989, J. Quant. Spectrosc. Radiat. Transfer, 42, 539

Guillot, T., Burrows, A., Hubbard, W. B., Lunine, J. I., \& Saumon, D. 1996, ApJ, 459, L35

Guillot, T., \& Havel, M. 2011, A\&A, 527, A20

Guillot, T., Santos, N. C., Pont, F., et al. 2006, A\&A, 453, L21

Guillot, T., \& Showman, A. P. 2002, A\&A, 385, 156

Hayward, T. L., Brandl, B., Pirger, B., et al. 2001, PASP, 113, 105

Hebb, L., Collier-Cameron, A., Loeillet, B., et al. 2009, ApJ, 693, 1920

Henry, G. W., Marcy, G. W., Butler, R. P., \& Vogt, S. S. 2000, ApJ, 529, L41

Holman, M. J., Winn, J. N., Latham, D. W., et al. 2006, ApJ, 652, 1715

Howell, S. B., Everett, M. E., Sherry, W., Horch, E., \& Ciardi, D. R. 2011, AJ, 142,19

Hubeny, I., Burrows, A., \& Sudarsky, D. 2003, ApJ, 594, 1011

Ibgui, L., \& Burrows, A. 2009, ApJ, 700, 1921

Jackson, B., Greenberg, R., \& Barnes, R. 2008, ApJ, 681, 1631
Jenkins, J. M., Borucki, W. J., Koch, D. G., et al. 2010a, ApJ, 724, 1108

Jenkins, J. M., Caldwell, D. A., Chandrasekaran, H., et al. 2010b, ApJ, 713, L120

Jenkins, J. M., Caldwell, D. A., Chandrasekaran, H., et al. 2010c, ApJ, 713, L87

Kipping, D. M. 2010, MNRAS, 408, 1758

Knutson, H. A., Charbonneau, D., Allen, L. E., Burrows, A., \& Megeath, S. T. 2008, ApJ, 673, 526

Knutson, H. A., Charbonneau, D., Cowan, N. B., et al. 2009, ApJ, 690, 822

Knutson, H. A., Howard, A. W., \& Isaacson, H. 2010, ApJ, 720, 1569

Koch, D. G., Borucki, W. J., Basri, G., et al. 2010, ApJ, 713, L79

Kovács, G., Bakos, G. Á., Hartman, J. D., et al. 2010, ApJ, 724, 866

Latham, D. W., Rowe, J. F., Quinn, S. N., et al. 2011, ApJ, 732, L24

Laughlin, G., Crismani, M., \& Adams, F. C. 2011, ApJ, 729, L7

Lissauer, J. J., Fabrycky, D. C., Ford, E. B., et al. 2011, Nature, 470, 53

Lodders, K. 1999, ApJ, 519, 793

Lodders, K. 2003, ApJ, 591, 1220

Lodders, K., \& Fegley, B. 2002, Icarus, 155, 393

Lodders, K., \& Fegley, B. 2006, Astrophysics Update 2 (Springer Praxis Books; Berlin: Springer)

Mandel, K., \& Agol, E. 2002, ApJ, 580, L171

Mandushev, G., O’Donovan, F. T., Charbonneau, D., et al. 2007, ApJ, 667, L195

Markwardt, C. B. 2009, in ASP Conf. Ser. 411, Astronomical Data Analysis Software and Systems XVIII, ed. D. A. Bohlender, D. Durand, \& P. Dowler (San Francisco, CA: ASP), 251

Marley, M. S., Fortney, J., Seager, S., \& Barman, T. 2007, in Protostars and Planets V, ed. B. Reipurth, D. Jewitt, \& K. Keil (Tucson, AZ: Univ. Arizona Press), 733

Marley, M. S., Gelino, C., Stephens, D., Lunine, J. I., \& Freedman, R. 1999, ApJ, 513,879

Marley, M. S., \& McKay, C. P. 1999, Icarus, 138, 268

Marley, M. S., Saumon, D., Guillot, T., et al. 1996, Science, 272, 1919

Marley, M. S., Seager, S., Saumon, D., et al. 2002, ApJ, 568, 335

McKay, C. P., Pollack, J. B., \& Courtin, R. 1989, Icarus, 80, 23

Miller, N., \& Fortney, J. J. 2011, ApJ, 726, L29

Miller, N., Fortney, J. J., \& Jackson, B. 2009, ApJ, 702, 1413

Pont, F., Zucker, S., \& Queloz, D. 2006, MNRAS, 373, 231

Press, W. H., Teukolsky, S. A., Vetterling, W. T., \& Flannery, B. P. 1992, Numerical Recipes in FORTRAN. The Art of Scientific Computing (Cambridge: Cambridge Univ. Press)

Saumon, D., \& Marley, M. S. 2008, ApJ, 689, 1327

Seager, S., \& Deming, D. 2010, ARA\&A, 48, 631

Seager, S., Whitney, B. A., \& Sasselov, D. D. 2000, ApJ, 540, 504

Showman, A. P., \& Guillot, T. 2002, A\&A, 385, 166

Sozzetti, A., Torres, G., Charbonneau, D., et al. 2009, ApJ, 691, 1145

Spiegel, D. S., \& Burrows, A. 2010, ApJ, 722, 871

Sudarsky, D., Burrows, A., \& Hubeny, I. 2003, ApJ, 588, 1121

Sudarsky, D., Burrows, A., \& Pinto, P. 2000, ApJ, 538, 885

Toon, O. B., McKay, C. P., Ackerman, T. P., \& Santhanam, K. 1989, J. Geophys. Res., 94, 16287

Torres, G., Fressin, F., Batalha, N. M., et al. 2011, ApJ, 727, 24

Troy, M., Dekany, R. G., Brack, G., et al. 2000, Proc. SPIE, 4007, 31

Vogt, S. S., Allen, S. L., Bigelow, B. C., et al. 1994, Proc. SPIE, 2198, 362

Werner, M. W., Roellig, T. L., Low, F. J., et al. 2004, ApJS, 154, 1

Winn, J. N., Holman, M. J., Torres, G., et al. 2008, ApJ, 683, 1076

Wright, J. T., Fakhouri, O., Marcy, G. W., et al. 2011, PASP, 123, 412

Wright, J. T., Upadhyay, S., Marcy, G. W., et al. 2009, ApJ, 693, 1084

Youdin, A. N., \& Mitchell, J. L. 2010, ApJ, 721, 1113 